IPMU11-0050

\title{
A Revisit to Top Quark Forward-Backward Asymmetry
}

\author{
Jing Shu ${ }^{a, b} *$ Kai Wang $a, b+i$ and Guohuai Zhu $d \oplus$ \\ a Zhejiang Institute for Modern Physics (ZIMP), \\ Zhejiang University, Hangzhou, Zhejiang 310027, CHINA \\ ${ }^{b}$ Institute for the Physics and Mathematics of the Universe (IPMU), \\ the University of Tokyo, Kashiwa, Chiba 277-8568, JAPAN
}

\begin{abstract}
We analyze various models for the top quark forward-backward asymmetry $\left(A_{F B}^{t}\right)$ at the Tevatron, using the latest CDF measurements on different $A_{F B}^{t} \mathrm{~s}$ and the total cross section. The axigluon model in Ref. [5] has difficulties in explaining the large rapidity dependent asymmetry and mass dependent asymmetry simultaneously and the parameter space relevant to $A_{F B}^{t}$ is ruled out by the latest dijet search at ATLAS. In contrast to Ref. [8], we demonstrate that the large parameter space in this model with a $U(1)_{d}$ flavor symemtry is not ruled out by flavor physics. The $t$-channel flavor-violating $Z^{\prime}, W^{\prime}$ and diquark models all have parameter regions that satisfy different $A_{F B}$ measurements within $1 \sigma$. However, the heavy $Z^{\prime}$ model which can be marginally consistent with the total cross section is severely constrained by the Tevatron direct search of samesign top quark pair. The diquark model suffers from too large total cross section and is difficult to fit the $t \bar{t}$ invariant mass distribution. The electroweak precision constraints on the $W^{\prime}$ model based on $Z^{\prime}-Z$ mixings is estimated and the result is rather weak $\left(m_{Z^{\prime}}>450 \mathrm{GeV}\right)$. Therefore, the heavy $W^{\prime}$ model seems to give the best fit for all the measurements. The $W^{\prime}$ model predicts the $t \bar{t}+j$ signal from $t W^{\prime}$ production and is $10 \%-50 \%$ of SM $t \bar{t}$ at the $7 \mathrm{TeV}$ LHC. Such $t+j$ resonance can serve as the direct test of the $W^{\prime}$ model.
\end{abstract}

\footnotetext{
* jing.shu@ipmu.jp

$\dagger$ kai.wang@ipmu.jp

$\ddagger$ zhugh@zju.edu.cn
} 
The prompt decay of top quark before hadronization provides opportunity to explore its various properties like charge, mass and spin. Given its large mass, the scale of top quark pair production is greater than $2 m_{t}$ where the perturbative QCD plays important role. Therefore, the top quark pair production at hadron colliders can serve as handle of precision test of the standard model (SM) gauge interaction, both weak interaction in its decay and the perturbative QCD theory of strong interaction in its production.

From the structure of the SM, top quark is special. As a colored particle, it is the heaviest known particle which is copiously produced at the hadron collider. Since the top quark acquire its large mass through the electroweak symmetry breaking (EWSB), any of its properties deviated from SM would be an important signals for new physics and potentially indicate the origin of EWSB, which makes searching new physics in the top quark sector extremely interesting at both Tevatron and LHC.

One important measurement for top quark in top quark pair production is the top forward backward asymmetry, which is equivalent to charge asymmetry under $\mathrm{CP}$ transformation 1]. For the SM production, it involves the high precision calculation of QCD. At $\mathcal{O}\left(\alpha_{s}^{3}\right)$, the bremsstrahlung amplitudes $q \bar{q} \rightarrow Q \bar{Q} g$ carry an odd power of color charge hence have an odd charge conjugation parity in the interference terms among initial states radiation and final state radiation diagrams. There is also interference between the box diagram of $\mathcal{O}\left(\alpha_{s}^{4}\right)$ with the LO diagram that contributes to the charge asymmetry.

CDF collaboration has recently updated the measurements on the total forward-backward asymmetry in top quark pair production with the semi-leptonic $t \bar{t}$ data with integrated luminosity of $5.3 \mathrm{fb}^{-1} 1$. The observed total asymmetry measured in the lab frame and the $t \bar{t}$ rest frame are

$$
\begin{array}{ll}
A_{F B}^{t}=0.150 \pm 0.050(\text { stat }) \pm 0.024(\text { syst }) & (p \bar{p} \text { rest frame }) \\
A_{F B}^{t}=0.158 \pm 0.072(\text { stat }) \pm 0.017(\text { syst }) & (t \bar{t} \text { rest frame })
\end{array}
$$

which corresponds to the SM prediction based on the NLO simulation, Monte Carlo for FeMtobarn processes (MCFM), $0.038 \pm 0.006$ (in lab) and $0.058 \pm 0.009$ in $t \bar{t}$ rest frame respectively [3]. These measurements have improved the previous results based on $3.2 \mathrm{fb}^{-1}$ of $A_{F B}^{p \bar{p}}(\cos \theta)=0.19 \pm 0.069$ and $A_{F B}^{t \bar{t}}(\Delta \eta)=0.24 \pm 0.014$. [3] 2

\footnotetext{
${ }^{1}$ The top quark forward-backward asymmetry has also been measured in the di-lepton channel as $A_{F B}=$ $0.42 \pm 0.15$ (stat) \pm 0.05 (syst) in the $t \bar{t}$ rest frame with $5.1 \mathrm{fb}^{-1}$ data [2].

${ }^{2}$ Note that the recent D0 measurement $A_{F B}^{t}=(8 \pm 4$ (stat) \pm 1 (syst) $) \%$ is based on top-pair events that
} 
More importantly, with the enlarged data sample, CDF collaboration has also released two distributional measurements. The most interesting result is the mass dependent forward backward asymmetry. The mass dependent forward backward asymmetry in the $t \bar{t}$ rest frame

$$
A_{F B}^{t \bar{t}}\left(M_{t \bar{t}}>450 \mathrm{GeV}\right)=0.475 \pm 0.112
$$

in comparison to the QCD correction prediction $0.088 \pm 0.013$. This $3.5 \sigma$ deviation may be a strong indication for physics beyond the SM. The second measurement is the rapidity dependent asymmetry, which is frame independent, as

$$
\begin{aligned}
& A_{F B}(|\Delta y|>1.0)=0.611 \pm 0.256 \\
& A_{F B}(|\Delta y|<1.0)=0.026 \pm 0.104 \pm 0.056
\end{aligned}
$$

in comparison to the MCFM SM prediction as $A_{F B}(|\Delta y|>1.0)=0.123 \pm 0.018$ and $A_{F B}(|\Delta y|<1.0)=0.039 \pm 0.006$.

The ratio of the parton level asymmetries in the two different frames, which differ by longitudinal boost, is

$$
\frac{A^{p \bar{p}}}{A^{t \bar{t}}}=0.95 \pm 0.41
$$

with the error corrected for the expected correlation across frames in the NLO QCD assumption. Even though the uncertainty is still large, this close to 1 central value implies that the top events which contribute to the asymmetry mostly lie in the forward-backward direction so the asymmetries are less dependent of the longitudinal boosts along the beam direction. This feature is also shown in the $\Delta \eta$ dependent asymmetry $A_{F B}^{t \bar{t}}(|\Delta y|>1.0)=0.611$ which shows that the asymmetric events are mostly due to events with larger rapidity difference $\left|\eta_{t}-\eta_{\bar{t}}\right|$.

On the other hand, the measurement of $t \bar{t}$ cross section $\sigma_{t \bar{t}}$, updated by the $4.6 \mathrm{fb}^{-1} \mathrm{CDF}$ result (with $m_{t}=172.5 \mathrm{GeV}$ ), is $\sigma_{t \bar{t}}^{\exp }=7.50 \pm 0.31$ (stat) \pm 0.34 (syst) \pm 0.15 (Z theory) pb which is in very good agreement with SM theory prediction of $\sigma_{t \bar{t}}^{\text {th }}=7.5_{-0.7}^{+0.5} \mathrm{pb}$ at NNLO 3 . Therefore, in order for new physics to generate large asymmetry without changing the total production cross section, the new physics contribution must interfere with the leading SM satisfy the experimental acceptance, which is uncorrected for effects from reconstruction or selection and can not be used to compare with the CDF results [4].

3 The latest NNLL calculation shows the $\sigma_{t \bar{t}}\left(m_{t}=173.1 \mathrm{GeV}\right)=6.30 \pm 0.19_{-0.23}^{+0.31} \mathrm{pb}$ [1] which is significantly lower than the experimental results. However, we still use the old SM predictions since we do not know $\sigma_{t \bar{t}}\left(m_{t}=172.5 \mathrm{GeV}\right)$ for the latest results. 
production of $u \bar{u}, d \bar{d} \stackrel{g}{\rightarrow} t \bar{t}$ as color octet exchange in $s$-channel. For instance, in order for the $s$-channel massive $Z^{\prime}$ to explain the asymmetry, there is no interference between $s$-channel color singlet exchange $u \bar{u}, d \bar{d} \stackrel{Z^{\prime}}{\rightarrow} t \bar{t}$ and QCD $u \bar{u}, d \bar{d} \stackrel{g}{\rightarrow} t \bar{t}$. The asymmetry events due to $Z^{\prime}$ will significantly enhance the total cross section $\sigma_{t \bar{t}}$ at the same time and this causes a strong tension between fitting of $A_{F B}$ and $\sigma_{t \bar{t}}$. This requirement implies that there are only two categories of candidate models to solve this anomaly.

- First category of models contain $s$-channel color octet vector boson but with parity violation at both $q-\bar{q}-G$ and $t-\bar{t}-G$ vertices [5] 8 ].

- Second category correspond to the $t$-channel exchange of light gauge boson of maximal flavor violation that couples initial state $u, d$ quark to the third generation $t$ quark. The large asymmetry can be generated via Rutherford singularity behavior [9 16] .

Both categories of models have their realizations in the beyond SM models. Given the updated measurements, especially the new distributional measurements, we discuss the current status of various models. In addition, the models may have other implications that have been or will be constrained by some direct or indirect experiments. One realization of the first category models is the non-universal axigluon model proposed in [5] and it may receive constrain from low energy neutral meson mixings [8]. However, we show that the flavor bound can be easily evaded by putting a horizontal flavor symmetry $U(1)_{d}$. In the $W_{R}$ models [10, 14], since the $W_{R}$ is charged under SM $U(1)_{e m}$, the neutral component $W_{R}^{3}$ would inevitably mix with $W_{L}^{3}$ and some extra $U(1)_{X}$ which induce a $Z$ - $Z^{\prime}$ mixing. The new ATLAS Dijets [17] search and the Tevatron same sign dileptons [18] would severely constrain the s-channel axigluon models and the t-channel heavy $Z^{\prime}$ models. We also study the direct prediction at the Large Hadron Collider(LHC) using the $1 \sigma$ fitting of all three asymmetry measurements $A_{F B}^{t \bar{t}}\left(M_{t \bar{t}}>450 \mathrm{GeV}\right), A_{F B}^{t \bar{t}}(|\Delta y|>1.0), A_{F B}^{t \bar{t}}($ total) with the right total cross section.

The paper is organized as follows. In Section —, we presented the $1 \sigma$ fitting of all three asymmetry measurements for s-channel color octet model (Section IA), t-channel $Z^{\prime}$ model (Section [B 1), $W^{\prime}$ model (Section IB 2), diquark model (Section IB 3) and the corresponding consequences. In Section II, we calculate the production rates for the new particles in various different models at the Tevatron which give the bounds for those models and the LHC signals. In Section III, we consider some indirect bounds for the axigluon 
from flavor physics (Section ஹA) and $W^{\prime}$ model from electroweak precision test (EWPT) (Section ஹIB). Section [V contains our conclusions.

\section{UPDATED STATUS OF THE MODELS}

In this section, we discuss the updated status of the models based on the latest measurements, especially the new distributional measurements. The simulation in the following discussion is at parton level and leading order. The asymmetry observables are defined at parton level without taking into account possible reconstruction efficiency. The SM contribution to the asymmetries from MCFM simulation have been subtracted to the corresponding measured values. The total cross section is obtained by multiplying a QCD $k$-factor. Since the latest experimental value is based on $m_{t}=172.5 \mathrm{GeV}$, for better comparison, we employ the theory calculation at $\mathrm{NNLO}$ for $m_{t}=172.5 \mathrm{GeV}$ and the $k$-factor is 1.3 . Last, the differential cross section of $t \bar{t}$ invariant mass is not included as requirement in the scan since QCD correction [19] and cut efficiency [20] may significantly modify the shape of differential distribution $d \sigma / d_{M_{t \bar{t}}}$.

In the following discussion, we are mostly interested in the region where the three asymmetry measurements can be explained within $1 \sigma$.

\section{A. s-channel color octet}

The interference term between the color octet $V-A$ gauge boson $G_{\mu}^{a}$ contribution and gluon contribution in $q \bar{q}$ annihilation pickup a term as

$$
\frac{2 g_{s}^{2} \hat{s}\left(\hat{s}-M_{G}^{2}\right)}{\left(\hat{s}-M_{G}^{2}\right)^{2}+M_{G}^{2} \Gamma_{G}^{2}}\left[+2 g_{A}^{q} g_{A}^{t} \beta \cos \theta\right]
$$

where $g_{s}$ is the strong coupling, $g_{A}^{q}$ is the axial component of the coupling between $G_{\mu}^{a}$ and light quarks $q$ and $g_{A}^{t}$ is that of the top quark. If the interference contribution is positive asymmetry, it requires that the axial coupling $g_{A}^{q} g_{A}^{t}<0$ is inevitable 4 .

The $s$-channel models can be realized in various context. The first realization is the axigluon models where $S U(3)_{c}$ color gauge symmetry is only a remnant of $S U(3)_{L} \times S U(3)_{R}$

${ }^{4}$ This non-universal gauge interaction potentially cause the violation of GIM mechanism thus may be constrained from flavor changing neutral current (FCNC) processes such as neutral meson mixings and we discuss its implications in the next section. 
broken by a bi-triplet scalar and another color octet with axial coupling become massive. However, to achieve the $g_{A}^{q} g_{A}^{t}<0$ requirement, the axigluon model has to be non-universal and one example is the 4-generation model proposed in [5]. Another realization is the models of extra dimension theory where massive color octet Kaluza-Klein (KK) gluon couple to the SM quarks in the chiral form as a result of fermion profiles [21]. The large $m_{t}$ naturally implies that the top quark and light quarks couple to $K K$ gluon in different way.

One interesting feature that was discussed in the axigluon model [5] is the mass dependent asymmetry. Due to the opposite contribution to asymmetry between the interference term and new physics squared term, the asymmetry is positive when the centre-of-mass energy is at intermediate energy but when it is close to the threshold, the asymmetry may become negative. This bending-over in correlation between asymmetry $A^{t}$ and the centre-of-mass energy $M_{t \bar{t}}$ had been shown in the latest CDF measurements, in both the measurement with finite bin sizes of $M_{t \bar{t}}$ and the measurement with below/above $M_{t \bar{t}}$ edge.

We use the axigluon model as one example to illustrate the feature of $s$-channel models in comparison with the updated measurement. Figure 1 shows the summary of best fit

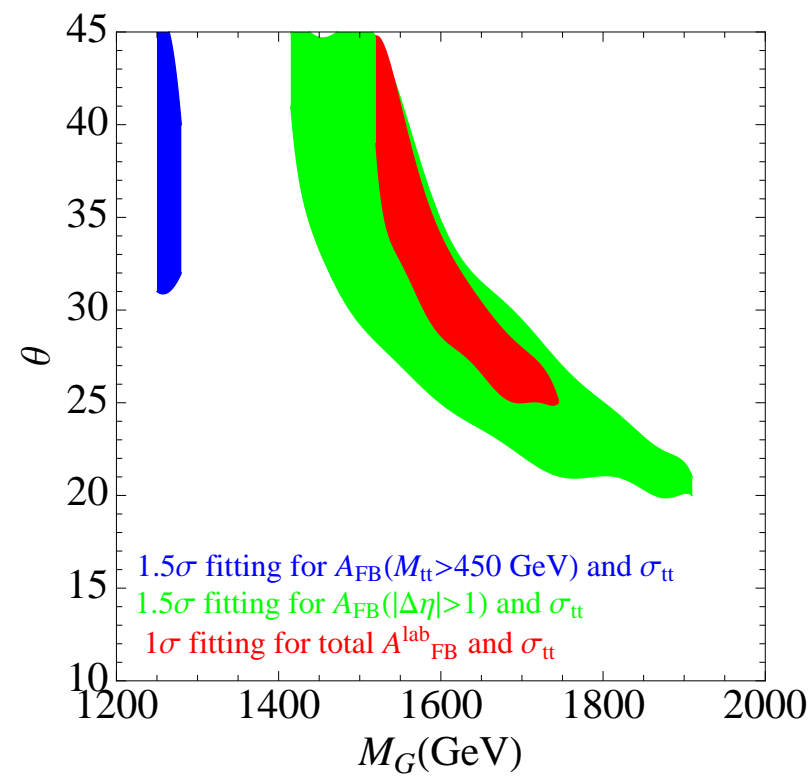

FIG. 1. $1 \sigma$ parameter region for constraints from the total asymmetry in the lab frame and the $t \bar{t}$ production rate; $1.5 \sigma$ parameter region for the $A_{F B}$ in $M_{t \bar{t}}>450$ and $|\Delta \eta|>1$ and $\sigma(p \bar{p} \rightarrow t \bar{t})$.

parameter regions for total asymmetry, mass dependent asymmetry, rapidity dependent asymmetry, the total cross section and the last bin of $d \sigma / d M_{t \bar{t}}$ measurements. Since the 
total asymmetry has been reduced from the previous fitting in [5], the $1 \sigma$ region with total asymmetry is enlarged as shown in Fig,1. However, there is no $1 \sigma$ region for the mass dependent asymmetry of $M_{t \bar{t}}>450 \mathrm{GeV}$ or the rapidity dependent asymmetry of $|\Delta \eta|>1$. Figure 1 shows the $1.5 \sigma$ parameter space for $A_{F B}(|\Delta \eta|>1), A_{F B}\left(M_{t \bar{t}}>450 \mathrm{GeV}\right)$ as well as the total $t \bar{t}$ production rate $\sigma(p \bar{p} \rightarrow t \bar{t})$. It is clearly shown that the axigluon model [5] does not consistently generate the large asymmetries in the events of $M_{t \bar{t}}>450 \mathrm{GeV}$ and $|\Delta \eta|>15$.

\section{B. t-channel}

As we argued, the ratio of $A^{p \bar{p}} / A^{t \bar{t}}$ close to one may imply that the top events are mostly in the forward-backward direction so the asymmetries are less dependent of the longitudinal boosts along the beam direction. Since the $t$-channel models naturally predict large number

of events in the forward-backward region, the close to one ratio of $A^{p \bar{p}} / A^{t \bar{t}}$ is a basic feature of $t$-channel models.

If the asymmetry is due to new physics in $t$-channel physics, the interference contribution between new physics and SM QCD is proportional to

$$
C_{F} \frac{g_{s}^{2} g_{N P}^{2}}{\hat{s} t_{t}}\left(u_{t}^{2}+\hat{s} m_{t}^{2}+\ldots\right)
$$

where $t_{t}=-\frac{1}{2} \hat{s}(1-\beta \cos \theta)$ and $1 / t$ expansion naturally picks up a $\cos \theta$. The $t$-channel physics naturally generates a large asymmetry in the $t \bar{t}$ system. In addition, the maximal asymmetry is generate at the Rutherford singularity where $\theta=0$ which corresponds to very high centre-of-mass energy. One would then expect the positive correlation between $A_{F B}^{t}$ and $M_{t \bar{t}}$.

The $t$-channel $Z^{\prime}$ model in [9, 12] proposed a color singlet neutral gauge boson with maximal flavor violation between first and third generations and the new contribution interferes with the SM $u \bar{u} \rightarrow t \bar{t}$. Similar to the $Z^{\prime}$ model, instead of neutral current exchange in $t$-channel, there is also a proposal using charge current exchange in $t$-channel as flavor violation $W^{\prime}$. The interference effect is reduced since it's only the $d \bar{d}$ initial state [10, 14]. Such

\footnotetext{
${ }^{5}$ One can use the general color octet vector boson with $V-A$ interaction to fit the two distributional asymmetries and total asymmetry [3]. The general results together with the most recent bounds in the dijet channel from ATLAS will be presented elsewhere.
} 
flavor violation gauge interactions may be realized in horizontal gauge interaction models [13] for neutral current or generalized left-right model [14] for charged current.

A Higgs-like scalar with maximal flavor violation[22] would generate a large negative asymmetry due to the helicity-flip in the Yukawa coupling. The spin conservation in the $\theta=0$ direction requires the top quark to move backward. To resolve this, the fermionnumber violating diquark scalars with maximal flavor violation was proposed [11, 15, 23]. Diquark scalar can be $3 \otimes 3=6 \oplus \overline{3}$ under $S U(3)_{c}$ and has fermion-number violating coupling as $\overline{t^{c}} u \phi$ or $\overline{t^{c}} d \phi$. Such diquark scalars with flavor violation can also be realized in various BSM contexts, partial unification models or supersymmetry. For instance, $R$ parity violation supersymmetric standard model which contains the baryon number violating coupling, $\epsilon_{\alpha \beta \gamma} u_{\alpha}^{c} d_{\beta}^{c} d_{\gamma}^{c}$ [24], the down type squark $\tilde{d}_{i}$ can mediated $u$-channel $d \bar{d} \rightarrow t \bar{t}$ that interferes with the QCD $d \bar{d} \rightarrow t \bar{t}$.

All the three proposals can in principle predict large positive asymmetry in $t \bar{t}$ production. In the following paragraphs, we examine the numerics to see whether the models can explain the three asymmetry measurements and the total cross section at the same time.

1. $Z^{\prime}$

We first examine the first proposed $t$-channel model, $Z^{\prime}$ [9]. To minimize the constraints from low energy, the authors proposed a right-handed coupled $Z^{\prime}$ with large coupling between $u$ and $t$. The parameter region for $1 \sigma$ fitting of all three asymmetry measurements as well as the total cross section for light $Z^{\prime}$ mostly below $t$ threshold is presented in Figure 2, Due to large destructive interference, the total cross section is always smaller than the measured value. This result is also shown in the NLO calculation of $Z^{\prime}$ model [19]. The best fit points for heavy $Z^{\prime}$ by requiring $1 \sigma$ fitting for all the three asymmetry measurements are listed in the Table $\rrbracket$. The corresponding $t \bar{t}$ cross section are also below the $1 \sigma$ total cross section and the best points are towards heavy masses of $\mathcal{O}(700 \mathrm{GeV})$.

One more complication which has been discussed in [9 11] is that the events in the t-channel exchange tend to be in high energy region which significantly increase the tail of $d \sigma / d M_{t \bar{t}}$, especially the last bin $(800 \mathrm{GeV}-1.4 \mathrm{TeV})$ in $d \sigma / d M_{t \overline{\bar{t}}}$. The QCD correction may change the shape and lower the contribution in high energy [19]. In addition, the $t$ channel kinematics implies that the top quark events at high energy are mostly in the larger 


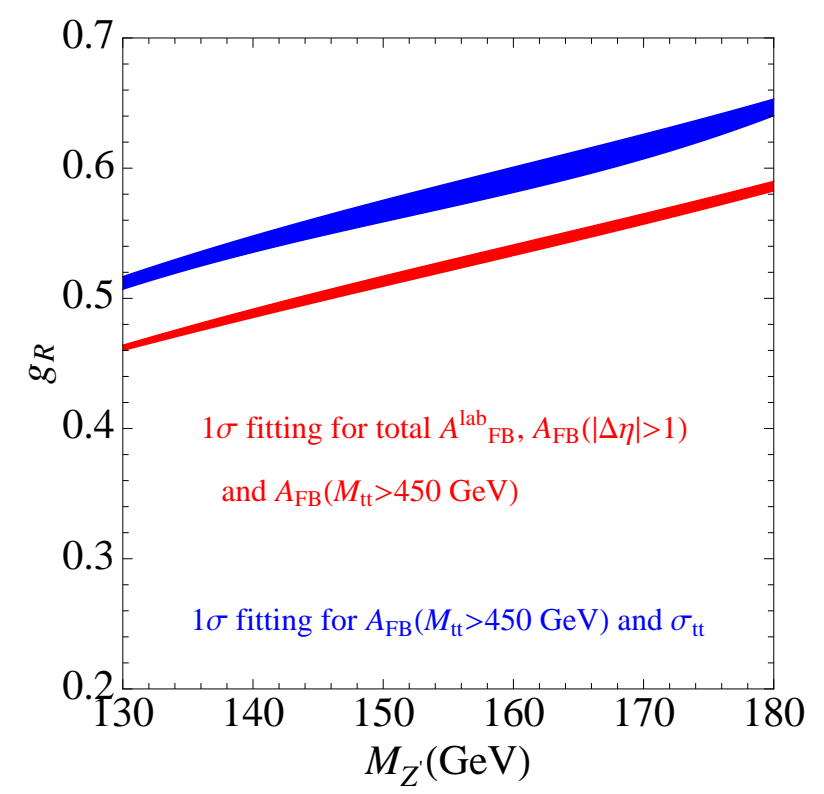

FIG. 2. Parameter space scan of $1 \sigma$ for all three asymmetries, $A_{F B}^{\text {total }}, A_{F B}(|\Delta \eta|>1)$ and $A_{F B}\left(M_{t \bar{t}}>450 \mathrm{GeV}\right)$ is shown in red. The $1 \sigma$ fitting for $A_{F B}\left(M_{t \bar{t}}>450 \mathrm{GeV}\right)$ and the total cross section $\sigma_{t \bar{t}}$.

\begin{tabular}{|c||c|c|c|c|}
\hline$M_{Z^{\prime}}, g_{R}$ & $A_{F B}^{\text {total }}$ & $A_{F B}\left(M_{t \bar{t}}>450 \mathrm{GeV}\right)$ & $A_{F B}(|\Delta \eta|>1)$ & $\sigma_{t \bar{t}}(\mathrm{pb})$ \\
\hline $275,0.8$ & $15.4 \%$ & $32.7 \%$ & $23.5 \%$ & 6.4 \\
$450,1.2$ & $15.8 \%$ & $34.4 \%$ & $23.4 \%$ & 6.6 \\
$575,1.5$ & $16.6 \%$ & $35.9 \%$ & $24.4 \%$ & 6.8 \\
$700,1.8$ & $16.7 \%$ & $36.1 \%$ & $24.7 \%$ & 6.9 \\
$750,1.9$ & $15.9 \%$ & $34.7 \%$ & $23.2 \%$ & 6.9 \\
\hline $\mathrm{CDF}$ & $5.7 \%-16.7 \%$ & $27.5 \%-50.0 \%$ & $23.1 \%-74.5 \%$ & $7.5 \pm 0.48$ \\
\hline
\end{tabular}

TABLE I. $1 \sigma$ benchmark points for all three asymmetry measurements. $k$-factor $=1.3, m_{t}=172.5$ $\mathrm{GeV}$ for $\sigma_{t \bar{t}}$.

rapidity region while the selection cut are more efficient for the central events. Consequently, the cut efficiency at high invariant mass is quite low [20], which may further decrease the effective total cross section. Polarization of top quark in the events sample also effect the cut efficiency. 


\section{2. $W^{\prime}$}

To resolve the tension between cross section and total asymmetry in the $Z^{\prime}$ model, the charged current process in $t$-channel may give better fit which has smaller interference effect due to the $d \bar{d}$ initial state. We plot the allowed parameter regions for the $t$-channel charged current model in [10] in Fig 3 .

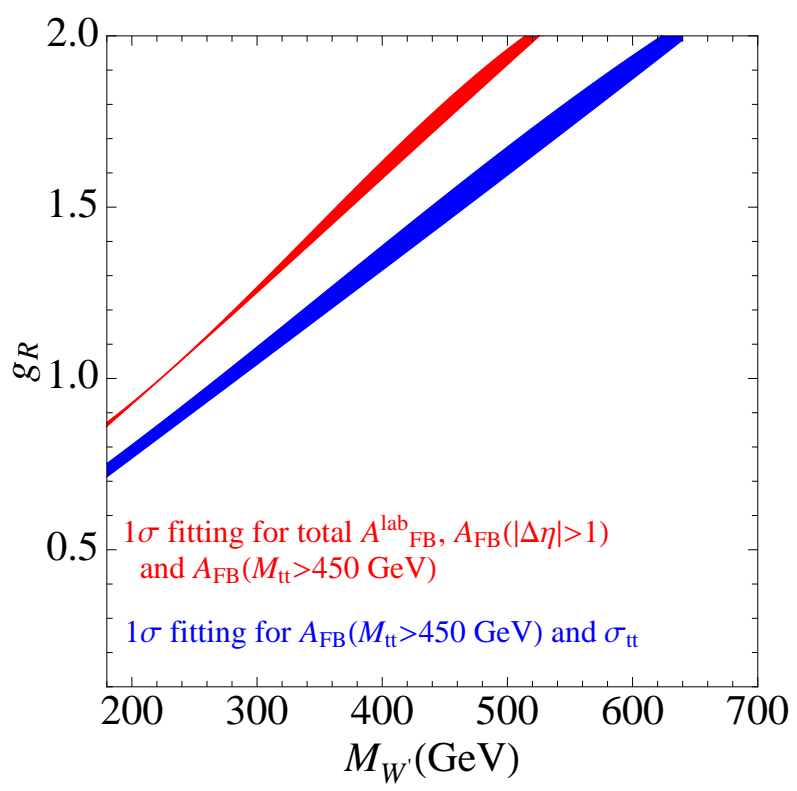

FIG. 3. Parameter space scan of $1 \sigma$ for all three asymmetries, $A_{F B}^{\text {total }}, A_{F B}(|\Delta \eta|>1)$ and $A_{F B}\left(M_{t \bar{t}}>450 \mathrm{GeV}\right)$ is shown in red. The $1 \sigma$ fitting for $A_{F B}\left(M_{t \bar{t}}>450 \mathrm{GeV}\right)$ and the total cross section $\sigma_{t \bar{t}}$.

The $1 \sigma$ asymmetry region of all three measurements corresponds to a larger total cross section which is outside the $1 \sigma$ fit of latest $\sigma_{t \bar{t}}$ measurement. However, various efficiency effects discussed in the last paragraph of $Z^{\prime}$ session may significantly reduce the measured cross section.

\section{Diquark}

We use the anti-triplet diquark that couples to $\overline{t^{c}} u \phi$ to illustrate the feature. Similar to the $W^{\prime}$ case, there also exist diquark scalars whose couplings are of $\overline{t^{c}} d \phi$ and these diquark scalars contribute to $d \bar{d} \rightarrow t \bar{t}$ instead. 
Figure 4 gives the $1 \sigma$ fitting for the anti-triplet diquark scalar with maximal flavor violation. The $1 \sigma$ region also exists for the anti-triplet diquark scalar for all the measurements

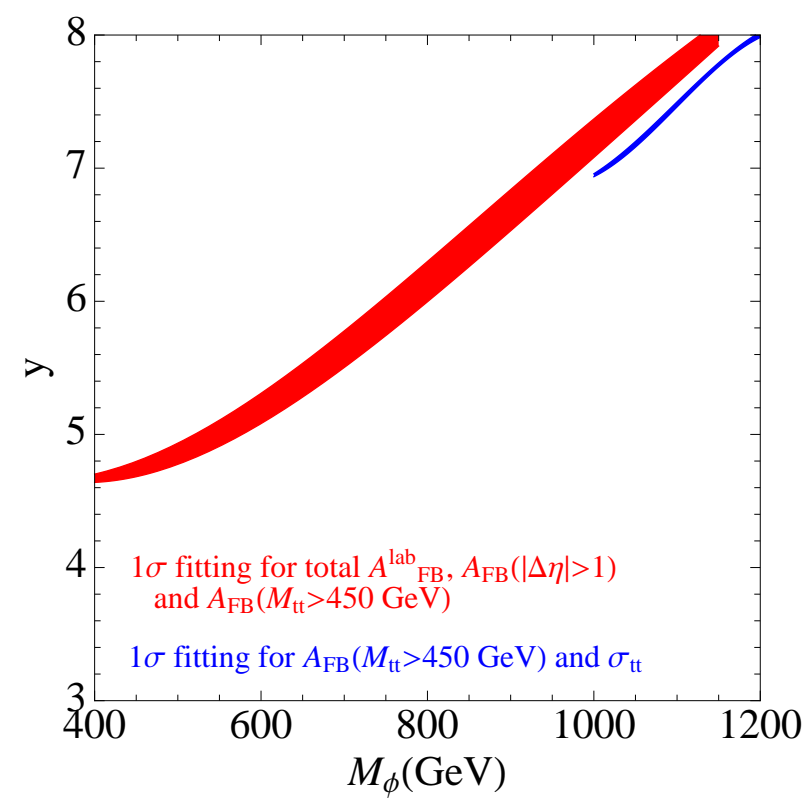

FIG. 4. Parameter space scan of $1 \sigma$ for all three asymmetries, $A_{F B}^{\text {total }}, A_{F B}(|\Delta \eta|>1)$ and $A_{F B}\left(M_{t \bar{t}}>450 \mathrm{GeV}\right)$ is shown in red. The $1 \sigma$ fitting for $A_{F B}\left(M_{t \bar{t}}>450 \mathrm{GeV}\right)$ and the total cross section $\sigma_{t \bar{t}}$.

in asymmetries $A_{F B}^{t}$. But the corresponding total cross section $\sigma_{t \bar{t}}$ are also larger than the measured value over $1 \sigma$. In addition, the best-fit for cross section and the mass dependent asymmetry is over $1 \mathrm{TeV}$ which makes the $d \sigma / d M_{t \bar{t}}$ measurement very difficult to fit as shown in [11]. The latest simulation by [20] also showed that the $t \bar{t}$ events generated by diquark scalar had a higher cut efficiency at high energy therefore the anti-triplet diquark fitting is worse than the $W^{\prime}$.

\section{IMPLICATIONS AT THE TEVATRON AND LHC}

After fitting the top forward backward asymmetries in different kinematical regions, we discuss the other Tevatron bounds for the models and the LHC predictions that can be soon tested in this section.

The Large Hadron Collider (LHC) is a proton-proton collider with centre-of-mass energy $7 \mathrm{TeV}$ in the first two years running. Unlike at Tevatron where the axigluon effect only 
appears as interference. The color octet axigluon of $\mathcal{O}(1 \mathrm{TeV})$ can be directly produced at the LHC and decay into dijet or $t \bar{t}$. With significant decay branching ratio (BR) to $t \bar{t}$, it provides additional handle to search it. The study of axigluon at the LHC has been performed by [7]. ATLAS collaboration has recently released the search for dijet resonance. The latest data has ruled out axigluon from 0.6-2.1 TeV by assuming axigluon coupling is only $g_{s}$. The axigluon model in [5] has a even larger coupling comparing with the ATLAS paper and therefore, the model receive much more server constraint.

For neutral gauge boson like $Z^{\prime}$, the flavor violating vertex of $u t$ will lead to large $u u \rightarrow t t$ or $\bar{u} \bar{u} \rightarrow \overline{t t}$ scattering with $Z^{\prime}$ exchange in the $t / u$-channel. The same-sign positive top quark pair $(u u \rightarrow t t)$ becomes particular interesting at the LHC given its large $u$-valence quark parton flux [25].

In addition, with large $u t$ coupling, the $t Z^{\prime}$ associate production is not negligible. Since $Z^{\prime}$ equally decays into $u \bar{t}$ and $t \bar{u}$, the associated production $t Z^{\prime}$ or $\bar{t} Z^{\prime}$ will contribute to $t t+j, \overline{t t}+j$ and $t \bar{t}+j$ final states. Again, since the LHC is proton proton collider, the $t t+j$ dominates the same-sign top production. The $t \bar{t}+j$ will appear in the inclusive $t \bar{t}$ search. Since the $1 \sigma$ parameter space of all the asymmetry constraints corresponds to smaller $t \bar{t}$ pair production, the additional $t \bar{t}+j$ may in principle help to ease the tension at Tevatron. However, if it significantly contribute to the $t \bar{t}$, the same amount of same-sign top quark will arise.

Figure 5 (a) gives the $p p \rightarrow t t$ production rate at Tevatron and the 7 TeV LHC with the $Z^{\prime}$ in the $1 \sigma$ fitting for all three asymmetry measurements. The $p \bar{p} \rightarrow t t+\overline{t t}$ at Tevatron is between $0.7-1 \mathrm{pb}$ for these best fit points. CDF measured only 3 events for $2 \mathrm{fb}^{-1}$ [18] with the acceptance range from $1.5 \%$ to $3 \%$. The best fit points all predict 15 -30 same-sign pure leptonic top events before selection cut but with one b-tagging. Even though these events from $t$-channel vector boson exchange may suffer from a low cut efficiency comparing to the $t$-channel light scalar exchange considered in Ref. [18], the $Z^{\prime}$ model is strongly constrained by the same-sign top quark scattering. At Tevatron, the same-sign top due to $t Z^{\prime}$ associate production is then much suppressed at the Tevatron due to significant phase space suppression.

The $u u \rightarrow t t$ scattering get significantly enhanced at the proton-proton collider LHC. The production rate can reach 200 pb. Therefore, even at very early running of LHC with about $30 \mathrm{pb}^{-1}$ data and requiring two b-tagging jet, the event number before kinematic cut 

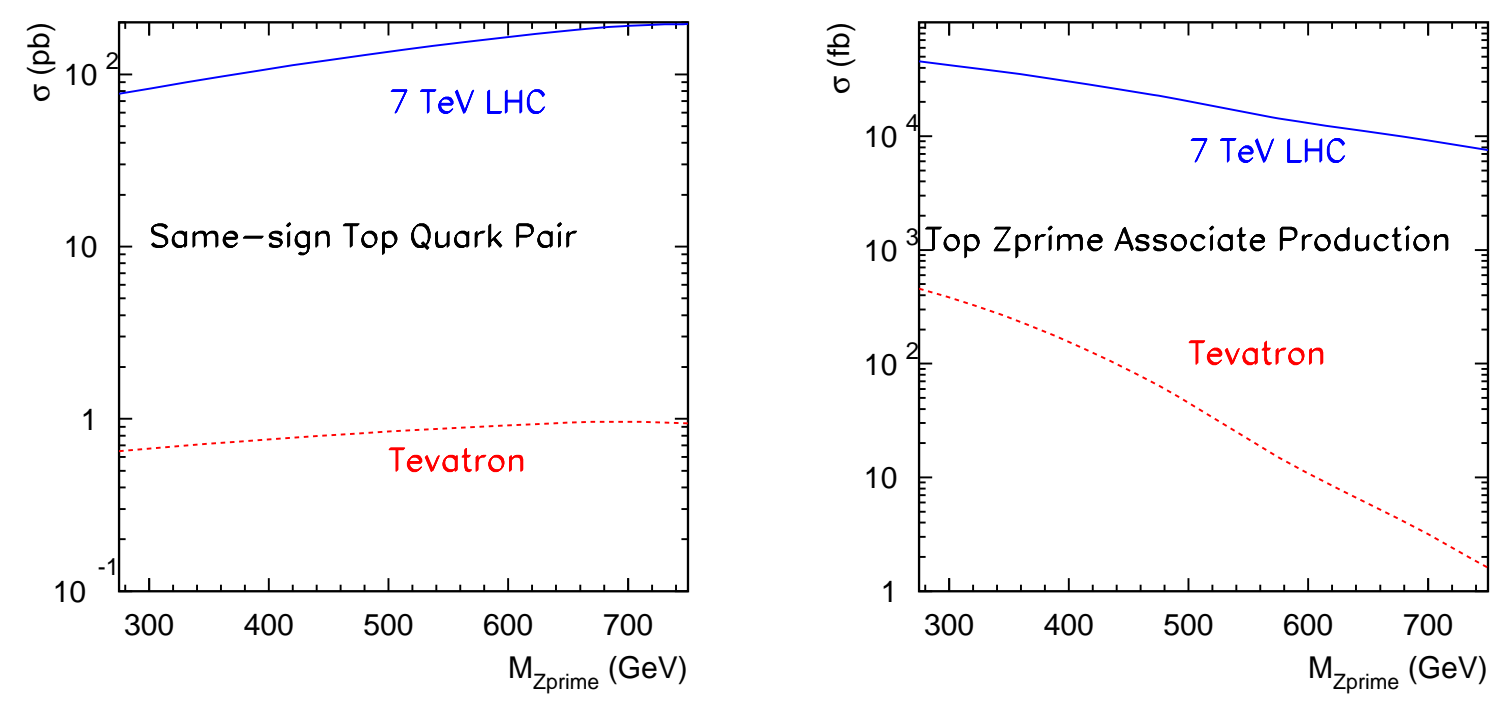

FIG. 5. (a) $\sigma(p p \rightarrow t t)$ at Tevatron and the $7 \mathrm{TeV}$ LHC; (b) $\sigma\left(p p \rightarrow t Z^{\prime}+\bar{t}+Z^{\prime}\right)$ at Tevatron and the $7 \mathrm{TeV}$ LHC. Both (a) and (b) are based on model parameter of $Z^{\prime}$ in the $1 \sigma$ fitting of all three asymmetry measurements as listed in Table —

is about 70 and the same-sign top quark $t t$ events is expected to be $\mathcal{O}(10)$.

For $W^{\prime}$ or diquark scalars with flavor violation, since they are electrically charged, it will only contribute to $t \bar{t}$ as at Tevatron. However, since the $W^{\prime}$ or diquark $\phi$ has a large $d t$ or $u t$ coupling, the $d g \rightarrow t W^{\prime}$ or $u g \rightarrow \bar{t} \phi$ production is significant as shown in [10, 11, 27]. With $W^{\prime}$ and diquark scalars of typically above top quark threshold, they can decay into $t$ plus one hard jet. The signal is then $t \bar{t}$ plus one hard jet and should appear in the inclusive $t \bar{t}$ searches. The diquark case has already been calculated in our early paper [11].

Figure 6 gives the production of top quark plus $W^{\prime}$ at hadron colliders. For $M_{W^{\prime}}$ lighter than $400 \mathrm{GeV}$, the production rate is about $0.1-1 \mathrm{pb}$ at the Tevatron. As we discussed in the previous section, the $1 \sigma$ fitting parameter space for all the asymmetries constraint corresponds to the larger cross section region. 6 The new contribution to $t \bar{t}+j$ will increase the tension between $A_{F B}$ and $\sigma_{t \bar{t}}$. For heavy $W^{\prime}$ above $400 \mathrm{GeV}$, due to large phase space suppression, the production rate at Tevatron can then be neglected. However, at the LHC, even with $7 \mathrm{TeV}$ centre-of-mass energy, the production rate is $\mathcal{O}(10 \mathrm{pb})$. Even though 6 [26] claims that the single top production at Tevatron puts a strong constraint on the $W^{\prime}-u-b$ coupling.

However this constraint does not apply to general $W^{\prime}$ models. 


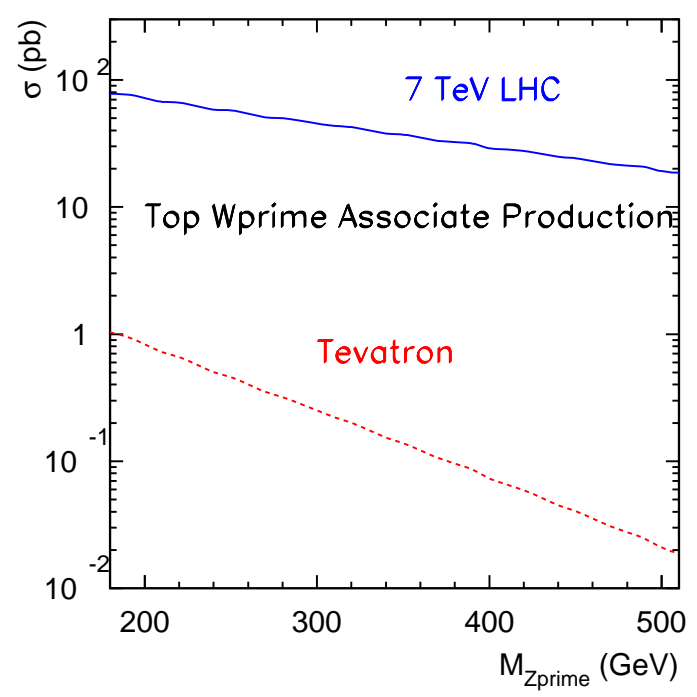

FIG. 6. $p p \rightarrow t W^{\prime} \rightarrow j+t \bar{t}$

$g g \rightarrow t \bar{t}$ dominates the $t \bar{t}$ at the LHC which does not interfere with $W^{\prime}$, the $t W^{\prime}$ itself may significantly increase the $t \bar{t}$ rate.

\section{INDIRECT CONSTRAINTS FOR MODELS}

\section{A. Axigluon with flavor protection}

As shown in the previous section, only non-universal axigluon models can provide the positive asymmetry. Being a color octet with strong coupling strength, this GIM violation axigluon will then lead to significant flavor changing neutral current (FCNC) effect.

$$
\mathcal{L}=i g_{L} \bar{q}^{i} \gamma^{\mu}\left(H_{L}^{q}\right)_{i j} P_{L} q^{j} T^{a} G_{\mu}^{a}+i g_{R} \bar{q}^{i} \gamma^{\mu}\left(H_{R}^{q}\right)_{i j} P_{R} q^{j} T^{a} G_{\mu}^{a}
$$

Flavor violation thus can arise from the non-universal gauge couplings due to the rotation between mass eigenstate and gauge eigenstate.

$$
u_{L}=V_{L}^{u} u_{L}, d_{L}=V_{L}^{d} d_{L}, u_{R}=V_{R}^{u} u_{R}, d_{R}=V_{R}^{d} d_{R}
$$

The effective coupling in horizontal space is

$$
V_{L}^{u \dagger} H_{L}^{u} V_{L}^{u}, V_{R}^{u \dagger} H_{R}^{u} V_{R}^{u}, V_{L}^{d^{\dagger}} H_{L}^{d} V_{L}^{d}, V_{R}^{d^{\dagger}} H_{R}^{d} V_{R}^{d},
$$


The rotation from mass eigenstate to gauge eigenstate for up and down type quark respectively is completely unmeasurable in the weak interaction. The only observable is the mixing in charge current transition which is categorized as CKM matrix 7 .

To avoid flavor violation in the down sector, one may introduce a $U(1)_{d}$ symmetry [28] which acts only on the down sector with different eigenvalues for different generations, but does not distinguish the handedness of the quarks. Then the down-quark sector are diagonal with $V_{L}^{d}=V_{R}^{d}=\mathbb{1}$ so that there is no FCNC at all in the $B_{s}, B_{d}$ or neutral $K$ system. For simplicity, we take further the rotation matrix of right-handed up-quark sector as $V_{R}^{u}=\mathbb{1}$. Then one can explicitly determine the left-handed up-type quark rotation based on the known CKM matrix using $V_{L}^{u} V_{L}^{d^{\dagger}}=V_{C K M}$

$$
V_{L}^{u}=V_{C K M}
$$

Nowadays the Wolfenstein parametrization [29] is widely used to express the CKM matrix in terms of four parameters $(\lambda, A, \rho$ and $\eta)$. To keep the unitarity of CKM matrix to all orders of $\lambda$, we adopt in the following a definition of Wolfenstein parameters proposed in [30]. Then the effective coupling between up quark and charm quark is

$$
\left(V_{L}^{u \dagger} H_{L}^{u} V_{L}^{u}\right)_{12}=-A^{2} \lambda^{5}(i \eta-\rho+1) .
$$

Under the assumption of above rotations, the FCNC operators only arise in left-handed and mixing between first and second generation up-type quarks as

$$
-\frac{1}{6}\left(\bar{u}_{L}^{\alpha} \gamma^{\mu} c_{L}^{\alpha}\right)\left(\bar{u}_{L}^{\beta} \gamma_{\mu} c_{L}^{\beta}\right)+\frac{1}{2}\left(\bar{u}_{L}^{\alpha} \gamma^{\mu} c_{L}^{\beta}\right)\left(\bar{u}_{L}^{\beta} \gamma_{\mu} c_{L}^{\alpha}\right),
$$

where the following decomposition satisfied by the color $\mathrm{SU}(3)$ fundamental representation has been implemented

$$
T_{\alpha \beta}^{a} T_{\gamma \epsilon}^{a}=\frac{1}{2} \delta_{\alpha \epsilon} \delta_{\beta \gamma}-\frac{1}{6} \delta_{\alpha \beta} \delta_{\gamma \epsilon} .
$$

Under Fierz transformation

$$
\left(\bar{u}_{L}^{\alpha} \gamma^{\mu} c_{L}^{\beta}\right)\left(\bar{u}_{L}^{\beta} \gamma_{\mu} c_{L}^{\alpha}\right)=\left(\bar{u}_{L}^{\alpha} \gamma^{\mu} c_{L}^{\alpha}\right)\left(\bar{u}_{L}^{\beta} \gamma_{\mu} c_{L}^{\beta}\right),
$$

the effective $\Delta C=2$ Hamiltonian can be expressed as

$$
H_{A G}^{\Delta C=2}=C(\mu)\left(\bar{u}_{L}^{\alpha} \gamma^{\mu} c_{L}^{\alpha}\right)\left(\bar{u}_{L}^{\beta} \gamma_{\mu} c_{L}^{\beta}\right)
$$

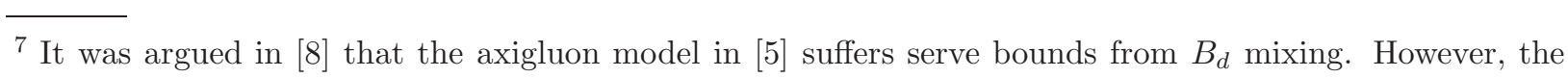
calculation seems to be done by assuming both up and down quark sectors transform like CKM rotation. 
and the leading order Wilson coefficient at the scale $m_{G}$ is

$$
C\left(m_{G}\right)=\frac{g^{2} A^{4} \lambda^{10}(1-\rho+i \eta)^{2}}{3 m_{G}^{2}} .
$$

It means that the $D^{0}-\bar{D}^{0}$ mixing in this axigluon model has $\lambda^{10}$ suppression due to CKM rotation. The RGE running of the above Wilson coefficient is well known 8 ,

$$
C\left(\mu_{c}\right)=\left(\frac{\alpha_{s}\left(m_{G}\right)}{\alpha_{s}\left(\mu_{c}\right)}\right)^{6 / 23} C\left(m_{G}\right)
$$

Notice that the hadronic matrix element of $\Delta C=2$ operator is

$$
\left\langle\bar{D}^{0}\left|\bar{u}_{L}^{\alpha} \gamma^{\mu} c_{L}^{\alpha} \bar{u}_{L}^{\beta} \gamma_{\mu} c_{L}^{\beta}\right| D^{0}\right\rangle \equiv \frac{2}{3} f_{D}^{2} m_{D}^{2} B_{D}\left(\mu_{c}\right)
$$

Just like the $B^{0}-\bar{B}^{0}$ mixing case, one may define the renormalization group invariant parameter $\hat{B}_{D}$ by

$$
\hat{B}_{D} \equiv\left(\alpha_{s}\left(\mu_{c}\right)\right)^{-6 / 23} B_{D}\left(\mu_{c}\right)
$$

which should be $O(1)$. Then the axigluon induced $\Delta C=2$ effective operator contributes to mass difference of neutral D system as

$$
\Delta m_{D}=\alpha_{s}\left(m_{G}\right)^{6 / 23} \frac{8 \pi f_{D}^{2} m_{D} \hat{B}_{D}}{9} \frac{\alpha_{s}\left(m_{G}\right) A^{4} \lambda^{10}\left((1-\rho)^{2}+\eta^{2}\right)}{m_{G}^{2}} .
$$

The axigluon model can also induce $\Delta C=1$ effective operator which would in principle affect $D^{0}-\bar{D}^{0}$ mixing by

$$
\left(M-\frac{i}{2} \Gamma\right)_{12}=\frac{1}{2 m_{D}}\left\langle\bar{D}^{0}\left|H_{e f f}^{\Delta C=2}\right| D^{0}\right\rangle+\frac{1}{2 m_{D}} \sum_{n} \frac{\left\langle\bar{D}^{0}\left|H_{e f f}^{\Delta C=1}\right| n\right\rangle\left\langle n\left|H_{e f f}^{\Delta C=1}\right| D^{0}\right\rangle}{m_{D}-E_{n}+i \epsilon} .
$$

Actually the experimental observation of comparably large mass and width differences [31]

$$
x \equiv \frac{\Delta m_{D}}{\Gamma_{D}}=0.98_{-0.26}^{+0.24 \%}, \quad y \equiv \frac{\Delta \Gamma_{D}}{2 \Gamma_{D}}=(0.83 \pm 0.16) \%
$$

strongly implies that they are dominated by the long distance effects of the SM $\Delta C=1$ operators. Therefore the axigluon induced $\Delta C=1$ terms could be safely neglected as they should be much smaller than the tree-level SM $\Delta C=1$ terms.

Taking the Wolfenstein parameters as [32]

$$
A=0.812, \quad \lambda=0.2254, \quad \rho=0.148, \quad \eta=0.351
$$

\footnotetext{
8 The RGE running is actually dependent on $N_{f}$, the number of active flavor via $\beta_{0}=11-2 N_{f} / 3$. From the scale $m_{G}$ down to $\mu_{c}, N_{f}$ changes correspondingly from 6 to 4 . But numerically this effect is small and we will simply take $N_{f}=5$ in the RGE running.
} 
and $f_{D}=207 \mathrm{MeV}[31]$, we obtain

$$
\left(\frac{\Delta m_{D}}{\Gamma_{D}}\right)_{\text {axigluon }}=0.082 \%\left(\frac{1 \mathrm{TeV}}{m_{G}}\right)^{2}\left(\frac{\hat{B}_{D}}{1}\right)
$$

which is roughly one order of magnitude smaller than the experimental result.

\section{B. Electroweak constrains on the $W^{\prime}$ model}

In general, the $W^{\prime}$ must generate its mass through gauge symmetry breaking, then some other neutral component in the $W^{\prime}$ symmetry breaking sector (for instance $W_{R}^{3}$ in the $S U(2)_{R}$ symmetry breaking) would inevitably mix with $W_{L}^{3}$ and some extra $U(1)$ so that the $W^{\prime \pm}$ would be charged under $U(1)_{\mathrm{em}}$. As a consequence, there is a large $Z$ - $Z^{\prime}$ mixing which is constrained by the electroweak precision test. In general, the bound from EWPT is subtle since different fermions $W / Z$ boson couplings are modified in different ways which may even depends on models and a careful global fit is needed. The full results for the $W^{\prime}$ model to explan the top forward backward asymmetry will be presented elsewhere. Since the overall modification for fermion $Z$ boson coupling is small (except for some right-handed quarks charged under $S U(2)_{R}$ ), we only consider the tree-level $Z-Z^{\prime}$ mixing as a rough estimation. For observables that strongly depends on $u / d / b_{R^{-}} Z$ coupling, such as $g_{R}^{2}, Q_{W}(C s)$, etc., their deviations from the SM results are still at the same level as $Z$ mass which is transmitted into $W$ boson mass.

We can start to consider a simple $S U(2)_{R} \times U(1)_{X} \times S U(2)_{L}$ model to estimate how large is the electroweak constraint for the $Z^{\prime}-Z$ mixing. The $S U(2)_{R}$ is separated from $S U(2)_{L}$ to avoid the troublesome $W^{\prime}-W$ mixing. The two double Higgs field $h_{L}$ and $h_{R}$ are charged under $S U(2)_{L} \times U(1)_{X}$ and $U(1)_{X} \times S U(2)_{R}$ respectively. The higgs fields get their vacuum expectation values $\left\langle h_{L}\right\rangle=u_{L}$ and $\left\langle h_{R}\right\rangle=u_{R}$ which spontaneously break $S U(2)_{R} \times U(1)_{X} \times S U(2)_{L}$ into the diagonal group $U(1)_{\mathrm{em}}$. In order to raise the $Z^{\prime}$ mass so we have less constrain from the $Z$ - $Z^{\prime}$ mixing, we choose $h_{R}$ transform as a triplet under $S U(2)_{R}$ so $m_{Z^{\prime}}=\sqrt{2} m_{W^{\prime}}$. The gauge quantum number for $h_{L}$ and $h_{R}$ are $(0,1,1 / 2)$ and $(1,2,0)$ under $S U(2)_{R} \times U(1)_{X} \times S U(2)_{L}$ respectively. For SM fermions, at least the quark doublet $(t, d)_{R}$ is charged under $S U(2)_{R}$ (It is possible to have some extra hidden fermions charged under $S U(2)_{R}$ and $U(1)_{X}$ to cancel the gauge anomaly). For the rest SM fermions, their quantum number is the same as the SM one if one replace their hyper charge as the 
$U(1)_{X}$ charge. The quantum number for $(t, d)_{R}$ and $(u, b)_{R}$ under $S U(2)_{R} \times U(1)_{X} \times S U(2)_{L}$ are $(1 / 2,1 / 3,0)$.

The kinetic term for the link fields $\operatorname{Tr}\left[\left(\mathrm{D}_{\mu} \mathrm{h}_{\mathrm{L}}\right)^{\dagger}\left(\mathrm{D}_{\mu} \mathrm{h}_{\mathrm{L}}\right)\right]+\operatorname{Tr}\left[\left(\mathrm{D}_{\mu} \mathrm{h}_{\mathrm{R}}\right)^{\dagger}\left(\mathrm{D}_{\mu} \mathrm{h}_{\mathrm{R}}\right)\right]$ becomes the mass terms for the massive gauge bosons. The mass matrix of the gauge bosons is

$$
\frac{1}{4}\left(\begin{array}{lll}
A_{\mu}^{R} & A_{\mu}^{X} & A_{\mu}^{L}
\end{array}\right)\left(\begin{array}{ccc}
2 g_{R}^{2} u_{R}^{2} & -2 g_{R} g_{X} u_{R}^{2} & 0 \\
-2 g_{R} g_{X} u_{R}^{2} & g_{X}^{2}\left(2 u_{R}^{2}+u_{L}^{2}\right) & -g_{X} g_{L} u_{L}^{2} \\
0 & -g_{X} g_{L} u_{L}^{2} & g_{L}^{2} u_{L}^{2}
\end{array}\right)\left(\begin{array}{c}
A_{\mu}^{R} \\
A_{\mu}^{X} \\
A_{\mu}^{L}
\end{array}\right) .
$$

We introduce the parameter $\epsilon \equiv u_{L}^{2} / 2 u_{R}^{2} \ll 1$ which shows that the right-handed symmetry breaking is only a perturbation.

This matrix can be diagonalized by means of an orthogonal matrix which we shall call R:

$$
\left(\begin{array}{c}
A_{\mu}^{R} \\
A_{\mu}^{X} \\
A_{\mu}^{L}
\end{array}\right)=\mathbf{R}^{\dagger}\left(\begin{array}{c}
A_{\mu} \\
Z_{\mu} \\
Z_{\mu}^{\prime}
\end{array}\right)
$$

where the mass eigenstates are denoted by $A, Z$, and $Z^{\prime}$. The eigenstate $A$ is massless and identified as the photon. The couplings of our theory are related to the electric charge by

$$
g_{R}=\frac{e}{\sin \phi \cos \theta_{W}}, \quad g_{X}=\frac{e}{\cos \phi \cos \theta_{W}}, \quad g_{L}=\frac{e}{\sin \theta_{W}}
$$

where $\theta_{W}$ is the weak mixing angle (in the limit $\epsilon \rightarrow 0$ ) and $\phi$ is an additional mixing angle. The other two eigenmasses are

$$
\begin{aligned}
& m_{Z}^{2}=\frac{1}{2} u_{L}^{2}\left(g_{Y}^{2}+g_{L}^{2}\right)\left[1-\epsilon \frac{g_{X}^{4}}{\left(g_{R}^{2}+g_{X}^{2}\right)^{2}}\right]=\frac{1}{2} u_{L}^{2}\left(g_{Y}^{2}+g_{L}^{2}\right)\left[1-\epsilon \sin ^{4} \phi\right], \\
& m_{Z^{\prime}}^{2}=\frac{1}{2} u_{R}^{2}\left(g_{R}^{2}+g_{X}^{2}\right)\left[1+\epsilon \frac{g_{X}^{4}}{\left(g_{R}^{2}+g_{X}^{2}\right)^{2}}\right]=\frac{1}{2} u_{R}^{2}\left(g_{R}^{2}+g_{X}^{2}\right)\left[1+\epsilon \sin ^{4} \phi\right],
\end{aligned}
$$

where we have dropped $\mathcal{O}\left(\epsilon^{2}\right)$ terms and

$$
\frac{1}{g_{Y}^{2}} \equiv \frac{1}{g_{R}^{2}}+\frac{1}{g_{X}^{2}}
$$

Clearly, $Z$ is identified with the SM $Z$ boson while $Z^{\prime}$ is referred to as the heavy $Z$ boson.

For small $\epsilon$, the mixing matrix $\mathbf{R}$ has the following approximate form:

$$
\mathbf{R}=\left(\begin{array}{ccc}
\sin \phi \cos \theta_{W} & \cos \phi \cos \theta_{W} & \sin \theta_{W} \\
\sin \phi \sin \theta_{W}+\epsilon \frac{\sin ^{3} \phi \cos ^{2} \phi}{\sin \theta_{W}} & \cos \phi \sin \theta_{W}-\epsilon \frac{\cos \phi \sin ^{4} \phi}{\sin \theta_{W}} & -\cos \theta_{W} \\
-\cos \phi+\epsilon \cos \phi \sin ^{4} \phi & \sin \phi+\epsilon \cos ^{2} \phi \sin ^{3} \phi & -\epsilon \cot \theta_{W} \cos \phi \sin ^{3} \phi
\end{array}\right),
$$




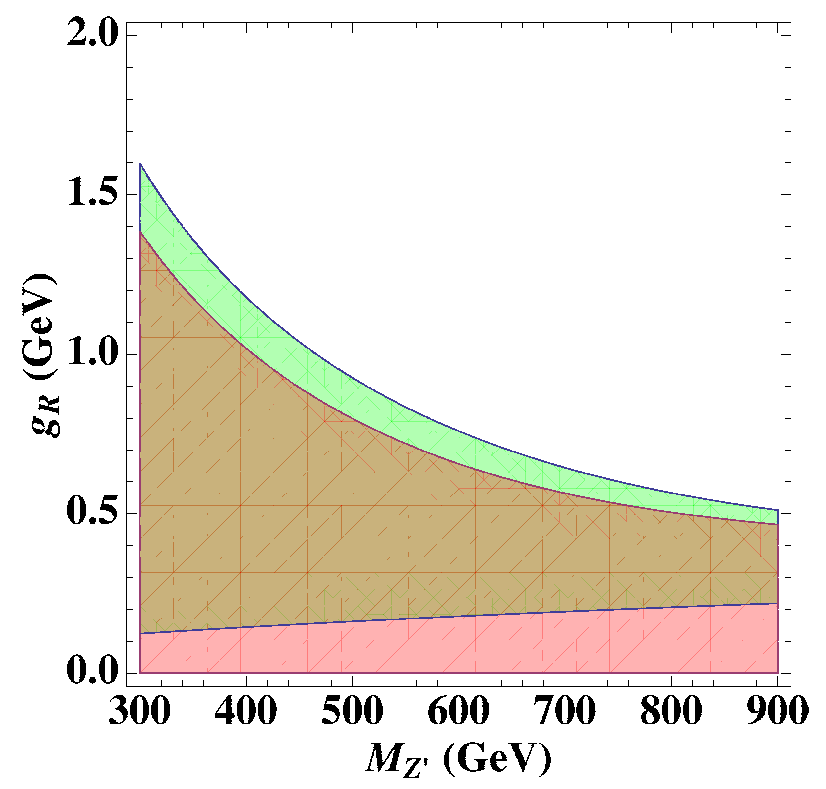

FIG. 7. The excluded region on the parameter spaces of $W_{R}$ model based on tree level contribution to $\mathrm{T}$ parameter alone from the new physics at $90 \% \mathrm{C}$. L. The green plus yellow region are is the excluded region for SM Higgs mass $m_{h}=114 \mathrm{GeV}$ while the red plus yellow region is the excluded region for SM Higgs mass $m_{h}=150 \mathrm{GeV}$.

from which it is simple to derive the SM fermion couplings. The SM fermion and Higgs couplings to $Z$ and $Z^{\prime}$ can be written as

$$
\begin{gathered}
g_{R} \sin \phi \sin \theta_{W} T_{R}^{3}+g_{X} \cos \phi \sin \theta_{W} Q_{X}-g_{L} \cos \theta_{W} T_{L}^{3} \\
=\frac{e}{\sin \theta_{W} \cos \theta_{W}}\left(\sin ^{2} \theta_{W} Q-T_{L}^{3}+\epsilon \sin ^{2} \phi \cos ^{2} \phi T_{R}^{3}-\epsilon \sin ^{4} \phi Q_{X}\right) \\
g_{R}\left(-\cos \phi+\epsilon \cos \phi \sin ^{4} \phi\right) T_{R}^{3}+g_{X}\left(\sin \phi+\epsilon \cos ^{2} \phi \sin ^{3} \phi\right) Q_{X}-g_{L}\left(-\epsilon \cot \theta_{W} \cos \phi \sin ^{3} \phi\right) T_{L}^{3} \\
=\frac{e}{\cos \theta_{W}}\left(-\frac{\cos \phi}{\sin \phi} T_{R}^{3}+\frac{\sin \phi}{\cos \phi} Q_{X}+\frac{\epsilon \cos \phi \sin ^{3} \phi}{\sin ^{2} \theta_{W}}\left(-T_{L}^{3}+Q \sin ^{2} \theta_{W}\right)\right) .
\end{gathered}
$$

In the limit of large $S U(2)_{R}$ breaking vev $(\epsilon \ll 1)$ and small mixings $(\phi \rightarrow 0)$, the Higgs current can be approximated as (we drop the $\epsilon \cos ^{2} \phi \sin ^{3} \phi$ term)

$$
J_{Z^{\prime}}^{\mu}(h)=-\frac{1}{2} g_{X} \sin \phi\left(h^{\dagger} D^{\mu} h\right)+h . c .,
$$

which induce a dimension six operator

$$
a_{h} \mathcal{O}_{h}=-\frac{g_{R}^{2}+g_{X}^{2}}{2 m_{Z^{\prime}}^{2}} \sin ^{4} \phi\left(h^{\dagger} D^{\mu} h\right)^{2} .
$$


which coincident with Eq. (29) that $\Delta m_{Z}^{2}=-\epsilon \sin \phi^{4} m_{Z}^{2}$. We can calculate the corresponding $\mathrm{T}$ parameter from the tree level gauge boson mixing,

$$
T=-\frac{a_{h} v^{2}}{\alpha_{f}}=\frac{\epsilon \sin ^{4} \phi}{\alpha_{f}}
$$

Using the SM model $m_{Z}, G_{F}$ (the life time of $\tau$ ) and $\alpha \equiv e^{2} / 4 \pi$ as the basic input parameter, we can calculate the allowed parameter space including the Higgs radiative corrections according to the most recent results: $S=0.03 \pm 0.09$ and $T=0.07 \pm 0.08$ (with $87 \%$ strong correlation) [31]. The results are presented in Fig 7. We can see that for sufficient heavy $Z^{\prime}$ and strong coupling $g_{R}$ (for instance, $g_{R}=2, m_{Z^{\prime}}=900 \mathrm{GeV}$ which is used in Ref. [20]), it is well above the excluded region.

\section{CONCLUSIONS}

We discuss the feature of various models for the top quark forward-backward asymmetry anomaly at Tevatron, using the latest CDF measurements on total asymmetry in lab frame $A_{F B}^{/ r m l a b}$, the rapidity dependent asymmetry $A_{F B}(|\Delta \eta|>1)$, the mass depedent asymmetry $A_{F B}\left(M_{t \bar{t}}>450 \mathrm{GeV}\right)$ and the total $t \bar{t}$ production cross section $\sigma_{t \bar{t}}$.

The axigluon model in [5] has difficulty in explain the large rapidity dependent asymmetry and the mass dependent asymmetry simultaneously. In addition, the latest dijet search [17] at ATLAS has ruled out the parameter region that is relevant to top $A_{F B}$. On the other hand, in contrast to the conclusion in Ref. [8], the model itself does not suffer from the flavor $B_{d}$ mixing under flavor protection $U(1)_{d}$ and a careful calculation shows that their to $D^{0}-\bar{D}^{0}$ is still one order lower than the current experimental bound.

The $t$-channel $Z^{\prime}$ [9], $W^{\prime}$ [10] and anti-triplet diquark [11] models all have parameter regions that satisfy all three asymmetry measurements within $1 \sigma$. However, the corresponding

production cross section predicted by the $1 \sigma$ asymmetry requirement in the $Z^{\prime}$ model are always significantly below the $1 \sigma$ of cross section measurement. The best fit point of $Z^{\prime}$ is about $700 \mathrm{GeV}$ with purely righthanded coupling $g_{u t}^{R} \simeq 1.8$ which corresponds to $6.9 \mathrm{pb}$. However, this best fit point will generate a large number of same-sign top quark events at Tevatron which is at least five times larger than the SM prediction. We conclude that the $Z^{\prime}$ model is very difficult to be consistent with all the measurements.

Both $W^{\prime}$ and anti-triplet diquark models predict the cross sections are larger than the measurement but various factors can lower the survival efficiency after cuts in these models 
to ease the tension between asymmetry and cross section. The best fit point for anti-triplet diquark lies in very high mass region and with better survival efficiency [20], it is difficult to fit the differential cross section $d \sigma / d M_{t \bar{t}}$. A rough estimation for $W^{\prime}$ model shows that the bounds from electroweak precision tests are weak due the heavy $Z^{\prime}$ and strongly coupling $g_{R}$. Therefore, we conclude that the best model is the $t$-channel $W^{\prime}$ model at the current stage. To test such model directly, we also use the $1 \sigma$ asymmetry parameters to compute the production rate of $t \bar{t}+j$ from $t W^{\prime}$ at $7 \mathrm{TeV}$ LHC and the production rate is $10 \%-50 \%$ of SM $t \bar{t}$.

Last, we want to mention that the latest NNLL calculation $\sigma_{t \bar{t}}\left(m_{t}=173.1 \mathrm{GeV}\right)=$ $6.30 \pm 0.19_{-0.23}^{+0.31} \mathrm{pb}[1]$ is significantly lower than the experimental results. If the result does not significantly change for $m_{t}=172.5 \mathrm{GeV}$ which is used for Tevatron experiments, then the fits for t-channel $W^{\prime}$ and anti-triplet would be better while the t-channel $Z^{\prime}$ would be worse.

\section{ACKNOWLEDGEMENT}

J.S. and K.W would like to thank Zhejiang Institute for Modern Physics at Zhejiang University and Prof. Mingxing Luo for hospitality after the Tohuku earthquake. We would like to thank Qinghong Cao, Mingxing Luo, Hitoshi Murayama, David Shih, Matt Strassler, Scott Thomas and Carlos Wagner for useful discussion. We also thank Tim Tait who initiate the electroweak bounds for $W^{\prime}$ model from our discussion. The work is partially supported by the World Premier International Research Center Initiative (WPI initiative) MEXT, Japan. J.S. and K.W. are also supported by the Grant-in-Aid for scientific research (Young Scientists (B) 21740169) and (Young Scientists (B) 22740143) from Japan Society for the Promotion of Science (JSPS), respectively. G.Z is supported in part by the National Science Foundation of China (No. 11075139 and No.10705024) and the Fundamental Research Funds for the Central Universities.

\section{NOTES ADDED}

While this work was being delayed by the huge earthquake in Japan, Ref. [20] appeared, which overlap with ours in the study of fitting different models on different top forward 
backward asymmetries. Our results agree with quantitatively with theirs for fitting different top forward backward asymmetries and the total $t \bar{t}$ cross section. However, we notice that the axigluon model and the heavy $Z^{\prime}$ model are severely constrained by the dijet search at the LHC (ATLAS) and the same sign dilepton search at the Tevatron (CDF). Therefore, we conclude that the heavy $W^{\prime}$ model is the most promising one at present. We also consider the indirect bounds for different models. 
[1] See for example, V. Ahrens, A. Ferroglia, M. Neubert, B. D. Pecjak, L. L. Yang, JHEP 1009, 097 (2010). arXiv:1003.5827 [hep-ph]]. L. G. Almeida, G. F. Sterman, W. Vogelsang, Phys. Rev. D78, 014008 (2008). arXiv:0805.1885 [hep-ph]]. M. T. Bowen, S. D. Ellis, D. Rainwater, Phys. Rev. D73, 014008 (2006). hep-ph/0509267].

[2] CDF collaboration, "Measurement of the Forward Backward Asymmetry in Top Pair Production in the Dilepton Decay Channel using $5.1 \mathrm{fb}^{-1}$ ", CDF Note 10436

[3] T. Aaltonen et al. [ CDF Collaboration ], arXiv:1101.0034 [hep-ex]].

[4] D $\emptyset$ Collaboration, "Measurement of the forward-backward production asymmetry of $\mathrm{t}$ and tbar quarks in $p \bar{p} \rightarrow t \bar{t}$ events", Conference Note D0 Note 6062-CONF

[5] P. H. Frampton, J. Shu and K. Wang, Phys. Lett. B 683, 294 (2010) arXiv:0911.2955 [hep$\mathrm{ph}]$.

[6] P. Ferrario, G. Rodrigo, Phys. Rev. D80, 051701 (2009). arXiv:0906.5541 [hep-ph]]. P. Ferrario, G. Rodrigo, JHEP 1002, 051 (2010). arXiv:0912.0687 [hep-ph]]. M. V. Martynov, A. D. Smirnov, arXiv:1006.4246 [hep-ph]]. M. Bauer, F. Goertz, U. Haisch, T. Pfoh, S. Westhoff, JHEP 1011, 039 (2010). arXiv:1008.0742 [hep-ph]]. C. -H. Chen, G. Cvetic, C. S. Kim, Phys. Lett. B694, 393-397 (2011). arXiv:1009.4165 [hep-ph]]. B. Xiao, Y. -k. Wang, S. h. Zhu, arXiv:1011.0152 [hep-ph]]. G. Burdman, L. de Lima, R. D. Matheus, Phys. Rev. D83, 035012 (2011). arXiv:1011.6380 [hep-ph]]. C. Degrande, J. -M. Gerard, C. Grojean, F. Maltoni, G. Servant, arXiv:1010.6304 [hep-ph]]. D. Choudhury, R. M. Godbole, S. D. Rindani, P. Saha, arXiv:1012.4750 [hep-ph]]. J. Cao, L. Wu, J. M. Yang, Phys. Rev. D83, 034024 (2011). arXiv:1011.5564 [hep-ph]]. R. Foot, arXiv:1103.1940 [hep-ph]].

[7] Y. Bai, J. L. Hewett, J. Kaplan, T. G. Rizzo, JHEP 1103, 003 (2011). arXiv:1101.5203 [hep-ph]].

[8] R. S. Chivukula, E. H. Simmons and C. P. Yuan, arXiv:1007.0260 [hep-ph].

[9] S. Jung, H. Murayama, A. Pierce, J. D. Wells, Phys. Rev. D81, 015004 (2010). arXiv:0907.4112 [hep-ph]].

[10] K. Cheung, W. -Y. Keung, T. -C. Yuan, Phys. Lett. B682, 287-290 (2009). arXiv:0908.2589 [hep-ph]].

[11] J. Shu, T. M. P. Tait, K. Wang, Phys. Rev. D81, 034012 (2010). arXiv:0911.3237 [hep-ph]]. 
[12] E. R. Barreto, Y. A. Coutinho, J. Sa Borges, Phys. Rev. D83, 054006 (2011). arXiv:1103.1266 [hep-ph]].

[13] S. Jung, A. Pierce and J. D. Wells, arXiv:1103.4835 [hep-ph].

[14] V. Barger, W. -Y. Keung, C. -T. Yu, Phys. Rev. D81, 113009 (2010). arXiv:1002.1048 [hep-ph]]. K. Cheung, T. -C. Yuan, arXiv:1101.1445 [hep-ph]]. J. Shelton, K. M. Zurek, arXiv:1101.5392 [hep-ph]]. V. Barger, W. -Y. Keung, C. -T. Yu, arXiv:1102.0279 [hep-ph]].

[15] A. Arhrib, R. Benbrik, C. -H. Chen, Phys. Rev. D82, 034034 (2010). arXiv:0911.4875] [hepph]]. I. Dorsner, S. Fajfer, J. F. Kamenik, N. Kosnik, Phys. Rev. D81, 055009 (2010). arXiv:0912.0972 [hep-ph]]. J. Cao, Z. Heng, L. Wu, J. M. Yang, Phys. Rev. D81, 014016 (2010). arXiv:0912.1447 [hep-ph]]. Z. Ligeti, M. Schmaltz, G. M. Tavares, arXiv:1103.2757 [hep-ph]].

[16] Q. -H. Cao, D. McKeen, J. L. Rosner, G. Shaughnessy, C. E. M. Wagner, Phys. Rev. D81, 114004 (2010). arXiv:1003.3461 [hep-ph]]. D. -w. Jung, P. Ko, J. S. Lee, S. -h. Nam, arXiv:1012.0102 [hep-ph]]. C. Delaunay, O. Gedalia, Y. Hochberg, G. Perez, Y. Soreq, [arXiv:1103.2297 [hep-ph]].

[17] ATLAS Collaboration, arXiv:1103.3864 [hep-ex].

[18] T. Aaltonen et al. [ CDF Collaboration ], Phys. Rev. Lett. 102, 041801 (2009). arXiv:0809.4903 [hep-ex]].

[19] B. Xiao, Y. -k. Wang, S. -h. Zhu, Phys. Rev. D82, 034026 (2010). arXiv:1006.2510 [hep-ph]].

[20] M. I. Gresham, I. -W. Kim, K. M. Zurek, arXiv:1103.3501 [hep-ph]].

[21] S. C. Park, J. Shu, Phys. Rev. D79, 091702 (2009). arXiv:0901.0720 [hep-ph]].

[22] S. Bar-Shalom, A. Rajaraman, D. Whiteson, F. Yu, Phys. Rev. D78, 033003 (2008). arXiv:0803.3795 [hep-ph]].

[23] J. M. Arnold, M. Pospelov, M. Trott, M. B. Wise, JHEP 1001, 073 (2010). arXiv:0911.2225 [hep-ph]].

[24] K. S. Babu, I. Gogoladze, K. Wang, Nucl. Phys. B660, 322-342 (2003). hep-ph/0212245.

[25] J. Cao, L. Wang, L. Wu, J. M. Yang, arXiv:1101.4456 [hep-ph]]. E. L. Berger, Q. -H. Cao, C. R. Chen, C. S. Li, H. Zhang, arXiv:1101.5625 [hep-ph]]. M. R. Buckley, D. Hooper, J. Kopp and E. Neil, arXiv:1103.6035 [hep-ph].

[26] N. Craig, C. Kilic and M. J. Strassler, arXiv:1103.2127 [hep-ph].

[27] M. I. Gresham, I. -W. Kim, K. M. Zurek, arXiv:1102.0018 [hep-ph]]. 
[28] C. Csaki, A. Falkowski and A. Weiler, Phys. Rev. D 80, 016001 (2009) arXiv:0806.3757 [hep-ph]].

[29] L. Wolfenstein, Phys. Rev. Lett. 51, 1945 (1983).

[30] A. J. Buras, M. E. Lautenbacher and G. Ostermaier, Phys. Rev. D 50, 3433 (1994) arXiv:hep-ph/9403384.

[31] K. Nakamura [Particle Data Group], J. Phys. G 37, 075021 (2010).

[32] J. Charles et al. [CKMfitter Group], Eur. Phys. J. C 41, 1 (2005) arXiv:hep-ph/0406184]; and updated results from http://ckmfitter.in2p3.fr. 